\title{
Optimisation fiabiliste d'une structure en alliage à mémoire de forme
}

\author{
Fatma Abid ${ }^{1,2, *}$, Abdelkhalak El Hami ${ }^{1}$, Tarek Merzouki ${ }^{3}$, Hassen Trabelsi ${ }^{2}$, Lassaad \\ Walha $^{2}$, Mohamed Haddar ${ }^{2}$ \\ ${ }^{1}$ Laboratoire de Mécanique de Normandie (LMN), Institut National des Sciences Appliquées de \\ Rouen, Rouen, France \\ ${ }^{2}$ Laboratoire de Mécanique, Modélisation et Productique (LA2MP), Département de Génie \\ Mécanique, Ecole Nationale d'Ingénieurs de Sfax, Sfax, Tunisie \\ ${ }^{3}$ Laboratoire de Génie des Systèmes de Versailles, Université de Versailles Saint Quentin en \\ Yvelines, VELIZY, France
}

\begin{abstract}
Résumé. L'objectif de l'optimisation fiabiliste (RBDO) est de trouver le meilleur compromis entre la sécurité et le coût. Plusieurs méthodes, telles que la méthode hybride (HM) et la méthode des facteurs optimaux de sûreté (OSF), sont développées pour atteindre cet objectif. Ces méthodes ont été appliquées uniquement pour les matériaux usuels. Néanmoins, le progrès effectué dans le domaine de l'élaboration de matériaux a conduit à l'utilisation des matériaux "intelligents" tel qua l'alliage à mémoire de forme (SMA). Dans ce papier, nous proposons une extension de ces méthodes dans le cas des structures formées en SMA pour étudier l'efficacité des méthodes proposées.
\end{abstract}

\section{Introduction}

Le développement au niveau informatique a permis de traiter des problèmes de plus en plus pointus dans des nombreux domaines tels que l'aéronautique, le biomédical, la production d'énergie ou encore l'automobile. La simulation des matériaux usuels a bénéficié de l'évolution rapide des approches numériques et de modélisation. Néanmoins, le progrès effectué dans le domaine de l'élaboration de matériaux à fort couplage multi physique a conduit à l'utilisation de matériaux "fonctionnels". Dans cette catégorie de matériaux dites aussi des matériaux intelligents ou avancés, les plus généralement utilisés sont ceux présentant un couplage magnétique/mécanique (magnétostrictif), électrique/ mécanique (piézo-électrique), ou encore thermique/mécanique (alliages à mémoire de forme) [1]. L'alliage à mémoire de forme (SMA) permet la conception des actionneurs ou de capteurs caractérisés par une grande simplicité $[2,3]$. Un tel matériau devient de plus en plus utilisé en raison de ses propriétés mécaniques comparées aux autres matériaux. Il est caractérisé par la capacité de se souvenir de sa forme originale après déformation. Ce comportement spécifique est dû à la capacité native de la structure cristallographique à subir des changements réversibles. Ces changements sont dus aux transformations martensitiques entre la cristallographie de la phase

\footnotetext{
Corresponding author: fatma.abid@insa-rouen.fr
} 
parentale la plus ordonnée, l'austénite, et la cristallographie de la phase parentale la moins ordonnée, la martensite. Les caractéristiques principales de cette catégorie de matériaux sont les capacités : (1) de récupérer la forme originale après une grande déformation induite par une charge mécanique (pseudo-élasticité) et (2) de récupérer des contraintes permanentes lors du chauffage (effet de mémoire de forme) [1]. Cependant, l'utilisation de tels matériaux nécessite d'améliorer sans cesse les techniques de conception des structures intelligents. Il est donc important de mettre à la disposition des ingénieurs des outils capables de dimensionner ces structures. L'optimisation intervient de façon primordiale dans l'augmentation des performances.

Cependant, les conceptions optimisés sans prise en considération de l'incertitude sur les paramètres peuvent ne pas respecter certains critères de fiabilité. La prise en compte de l'incertitude dans l'analyse mécanique est nécessaire pour un dimensionnement robuste et optimale. C'est avec cet objectif que les approches probabilistes sont développées. Ces méthodes permettent en effet d'un part d'étudier l'influence da la variabilité des paramètres sur le comportement de système et d'autre part, d'étudier sa fiabilité. La théorie de la fiabilité des structures consiste à définir la probabilité de défaillance d'une structure par la probabilité que les conditions de bon fonctionnement ne soient pas respectées. On appelle cette étude l'analyse de la fiabilité [4]. L'intégration de l'analyse de fiabilité dans les problèmes d'optimisation constitue une nouvelle discipline introduisant des critères de fiabilité dans la recherche de la configuration optimale des structures, c'est le domaine de l'optimisation fiabiliste (RBDO) [5]. Cette méthodologie de RBDO vise donc à considérer la propagation des incertitudes dans les performances mécaniques en s'appuyant sur une modélisation probabiliste des fluctuations des paramètres d'entrée. L'objectif principal de ce papier est donc de déterminer la meilleure méthodologie à suivre pour l'optimisation fiabiliste des structures formées par des matériaux intelligents.

\section{Optimisation déterministe (DDO)}

L'utilisation de l'approche d'optimisation déterministe (DDO) permet d'assigner des facteurs de sécurité dans les fonctions de contraintes du problème d'optimisation dans le but d'augmenter la sûreté de la conception et de compenser la présence des incertitudes dans les variables de conception. Le choix de ces facteurs de sécurité est basé sur l'expérience, les essais représentatifs ou bien la calibration issue d'un schéma probabiliste . Le problème d'optimisation déterministe peut être définir par:

$$
\begin{aligned}
& \min _{x} f(x) \\
& \text { tel que } \begin{cases}g_{k}(x) \leq 0 & k=1, \ldots, K \\
h_{j}(x)=0 & j=1, . ., L\end{cases}
\end{aligned}
$$

Où $x$ est le vecteur des variables de conception déterministes, $g_{k}(x)$ sont les contraintes d'inégalité et $h_{j}(x)$ sont les contraintes d'égalité. Le DDO offre une conception optimale. Cependant, il ne peut pas contrôler le niveau de fiabilité [6]. Par conséquent, l'optimisation fiabiliste (RBDO) est nécessaire pour garantir une conception fiable et optimale.

\section{Optimisation fiabiliste (RBDO)}

L'optimisation fiabiliste s'appuie donc sur l'analyse de la défaillance à chaque itération dans le but de déterminer les rôles des incertitudes sur le système et de concevoir des structures à la fois économiques et fiables. L'optimisation fiabiliste a été appliquée dans des différents domaines tels que : génie civile [10], mécatronique [11], vibro-acoustique [9, 
20], aérodynamique [12], biomécanique [13]. Un problème RBDO est considéré comme un problème d'optimisation sous des contraintes à la fois déterministes et probabilistes qui s'écrit sous la forme suivante:

$$
\begin{aligned}
& \min _{x} f(x) \\
& \text { tel que }\left\{\begin{array}{l}
\operatorname{Pr}\left[G_{i}(x, y) \leq 0\right] \leq P_{i}^{T} \quad i=1, . ., p \\
h_{j}(x) \leq 0 \quad j=p+1, . ., P
\end{array}\right.
\end{aligned}
$$

Avec $x$ est le vecteur des variables déterministes de la conception, $y$ est le vecteur des variables aléatoires, $\operatorname{Pr}\left[\right.$.] est l'opérateur de probabilité, $G_{i}$ est la fonction de performance, $P_{i}{ }^{T}$ est le niveau de fiabilité cible, $h_{j}$ est la contrainte déterministe, $p$ est le nombre des fonctions de performance et $P$ est le nombre total de contraintes. Il convient de noter que les variables de conception $x$ peuvent être des variables déterministes et représentent les paramètres de contrôle du système (par exemple matériaux, charges, dimensions) ou bien représentent la distribution probabiliste des paramètres (par exemple les valeurs moyennes et les écart-type des variables aléatoires $y$ ) [11]. Les variables aléatoires $y$ peuvent être des caractéristiques matérielles, des dimensions géométriques ou des chargements appliquées. Les incertitudes de ces variables peuvent être modélisées par des informations statistiques. La probabilité de défaillance est calculée par l'intégrale:

$$
\operatorname{Pr}\left[G_{i}(x, y) \leq 0\right\rfloor=\int \cdot \int_{G_{i}(x, y) \leq 0} f(y) d y
$$

Où $f_{y}(y)$ est la fonction de densité conjointe des variables aléatoires $y$. En tant que l'intégrale de l'équation (3) ne peut pas être calculé exactement, deux méthodes approximatives sont appliquées: les simulations stochastiques (Monte Carlo, ..) ainsi que les méthodes de moment (méthodes de fiabilité de premier et second ordre: FORM / SORM). Les méthodes de moment consistent à calculer l'indice de fiabilité, suivi d'une estimation de la probabilité de défaillance [11]. Ces méthodes sont basées sur la transformation des variables aléatoires dans l'espace physique aux variables gaussiennes indépendantes standards dans l'espace normalisé comme la Fig.1 le montre.

La transformation des variables aléatoires $y$ en les variables normalisées $u$ s'écrit:

$$
u=T(x, y) \leftrightarrow y=T^{-1}(x, u)
$$

Où l'opérateur T (.) est la transformation probabiliste.

Dans les applications pratiques, le couplage entre les procédures d'optimisation et l'analyse de fiabilité conduit à une faible stabilité de la convergence et à des temps de calcul très élevés. Il existe donc une motivation afin de développer des techniques efficaces pour réduire le temps de calcul. Dans le but d'améliorer la performance numérique, Kharmanda et al. [7,8] ont développé une méthode intitulée la méthode hybride. Celle ci consiste à résoudre le problème d'optimisation et de fiabilité simultanément. La solution est réalisée dans un espace hybride de conception (HSD) contenant les variables déterministes et aléatoires. La méthode hybride satisfait un niveau de fiabilité donné et réduit le temps de calcul. Cependant, le problème d'optimisation fiabiliste devient plus complexe que celui déterministe. Une autre méthode intitulée facteur de sureté optimale (OSF) $[14,15]$ a été élaborée. La solution optimale est obtenue en tenant compte du point de conception et des facteurs de sécurité calculés à partir de la dérivée de la fonction de contrainte. Cette méthode permet de réduire le temps de calcul par rapport à la méthode hybride. 


\section{1 Approche classique}

Généralement, en utilisant l'approche classique, nous utilisons deux espaces pour effectuer le problème RBDO. Ces espaces sont l'espace physique et l'espace normalisé [16]. Par conséquent, la solution optimale est obtenue en considérant deux problèmes imbriqués:

\section{i. Problème d'optimisation}

Elle consiste à minimiser une fonction objective $f(x)$ soumise à des contraintes déterministes $g_{k}(x)$ et à l'exigence de fiabilité. Il peut être défini par:

$$
\begin{aligned}
& \min _{x} f(x) \\
& \text { tel que }\left\{\begin{array}{l}
g_{k}(x) \leq 0 \quad k=1, . ., K \\
\beta(x, u) \geq \beta_{T}
\end{array}\right.
\end{aligned}
$$

Où $\beta(x, u)$ est l'indice de fiabilité de la structure et $\beta_{\mathrm{T}}$ est l'indice de fiabilité cible.

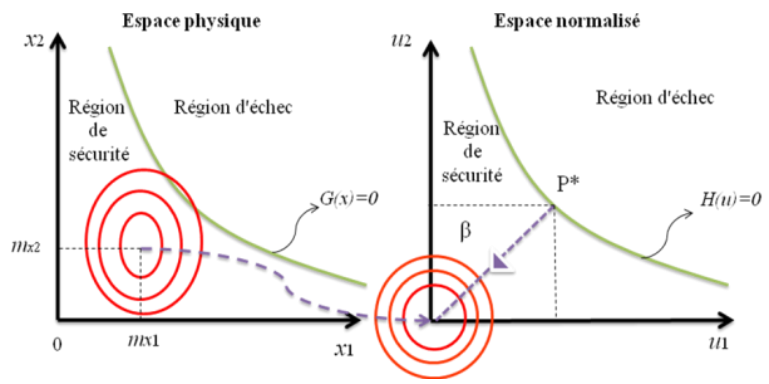

Fig. 1. Espace physique et espace normalisé

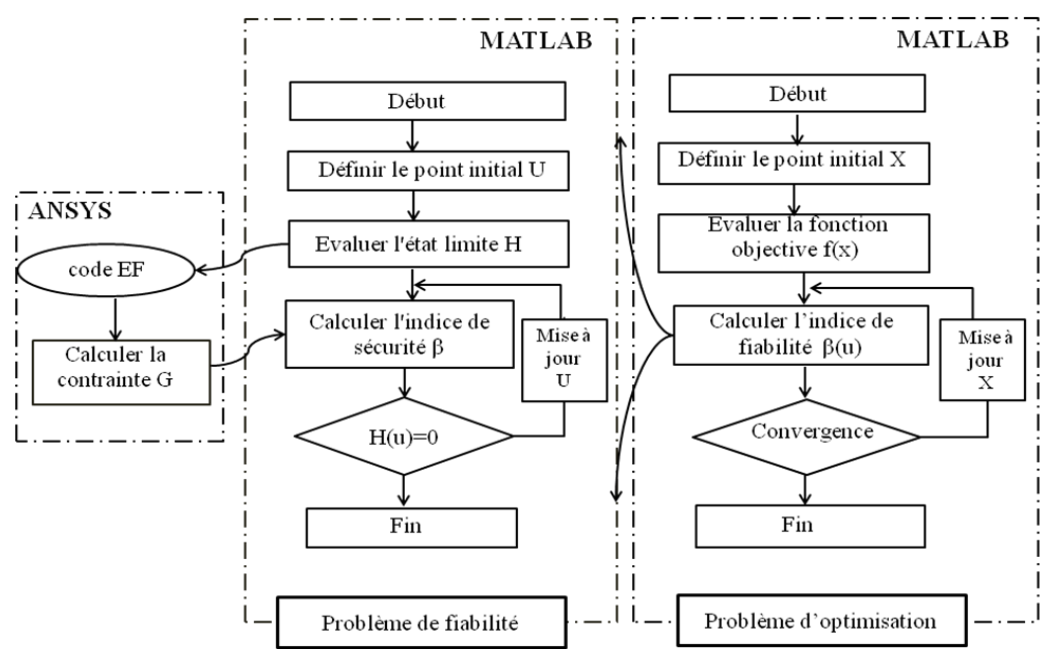

Fig. 2. Approche classique

\section{ii. Problème de fiabilité}

$\beta(x, u)$ représente la distance minimale entre l'origine et la fonction d'état limite $H(x, u)$ dans l'espace normalisé. Il peut être calculé en résolvant le problème de minimisation suivant: 


$$
\begin{aligned}
& \min _{x} d_{\beta}=\sqrt{\sum u_{i}^{2}} \\
& \text { tel que } H(x, u) \leq 0
\end{aligned}
$$

Il a été prouvé que l'approche classique nécessite un temps de calcul élevé en raison du nombre élevé de calculs numériques dans les deux espaces. Afin de réduire les effets de ce problème, une approche efficace appelée approche hybride a été élaborée [8].

\subsection{Méthode hybride}

L'approche hybride consiste à minimiser une nouvelle forme de la fonction objectif $F(x, y)$ sous les contraintes d'état limite et les contraintes déterministes, ainsi que des contraintes de fiabilité [8]. Le problème de la méthode hybride est écrit sous la forme:

$$
\begin{gathered}
\min _{x, y} F(x, y)=C(x) \times d_{\beta}(x, y) \\
\text { tel que }\left\{\begin{array}{l}
G(x, y) \leq 0 \\
g_{k}(x) \leq 0 \quad k=1, . ., K \\
\beta(x, u) \geq \beta_{t}
\end{array}\right.
\end{gathered}
$$

$d_{\beta}(x, y)$ présente la distance, dans l'espace hybride, entre le point optimal et le point de conception, $\beta(x, y)$ est l'indice de fiabilité de la structure et $\beta^{T}$ est l'indice de fiabilité cible. La minimisation de la fonction $F(x, y)$ est effectuée dans l'espace hybride de conception (HDS) des variables déterministes $x$ et des variables aléatoires $y$.

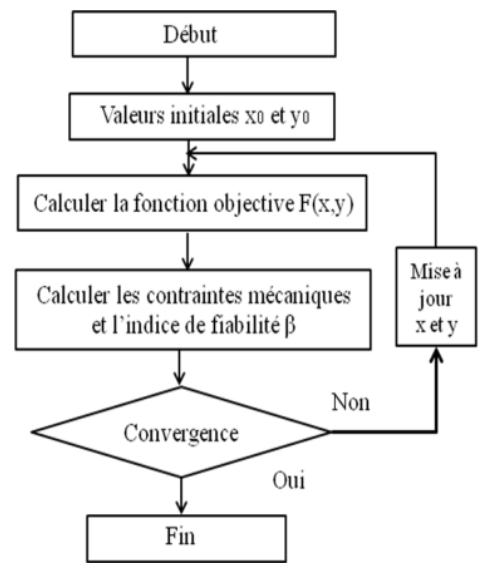

Fig. 3. Approche hybride

\subsection{Méthode des facteurs de sûreté optimaux (OSF)}

Dans l'approche d'optimisation déterminité (DDO), le choix du coefficient de sécurité est basé sur l'expérience de l'ingénieur et la solution obtenue ne peut pas satisfaire le niveau de fiabilité requis. Avec l'approche OSF, la détermination des facteurs de sécurité devrait être indépendante de l'expérience en ingénierie. Bien que la méthode hybride conduit à améliorer le niveau de fiabilité, l'approche OSF conduit à minimiser le temps de calcul. Elle consiste à utiliser l'analyse de sensibilité combinée à l'analyse de fiabilité afin d'éliminer les inconvénients de la méthode DDO et de la méthode hybride [17, 18$]$. 


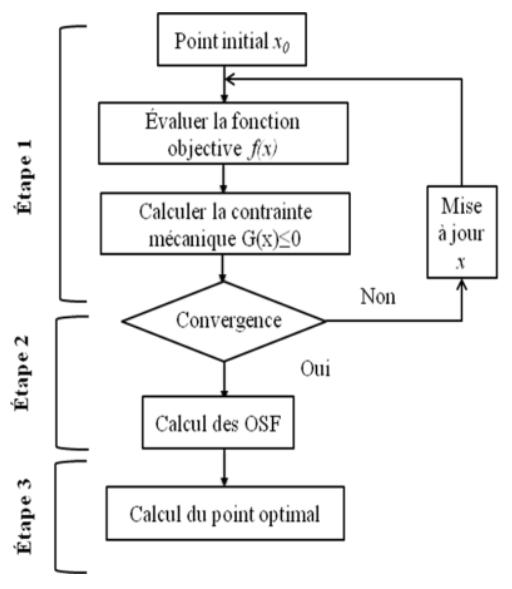

Fig. 4. Méthode des facteurs de sûreté optimaux

L'algorithme de la méthode OSF est composé des trois étapes principales :

\section{i- Détermination du point de conception $y_{i}^{*}$ :}

Nous minimisons ici la fonction objective soumise à la fonction d'état limite $G(y)$ et aux contraintes déterministes. La solution résultante est conçue par le point de défaillance le plus probable.

\section{ii- Calcul des facteurs de sécurité}

En fait, le point de conception $y_{i}^{*}$ est lié au point optimal $x_{i}^{*}$. Nous considérons ici que les valeurs moyennes de la variable aléatoire $m_{i}$ sont présentées par la variable déterministe $x_{i}$ [19].

$$
y_{i}^{*}=S_{f}^{i} x_{i}^{*}, i=1, . . n
$$

Pour une distribution normale de la variable aléatoire $y_{i}$, nous considérons la transformation dans l'espace normalisé (espace $u$ ) de l'espace physique (espace $x$ ) tel que :

$$
u_{i}=\frac{y_{i}-x_{i}}{\sigma_{i}}, i=1, . . n
$$

Les facteurs de sécurité peuvent être donc déterminés comme montré dans l'équation:

$$
S_{f}=1 \pm \gamma_{i} \beta_{t} \sqrt{\frac{\left|\frac{\partial G}{\partial y_{i}}\right|}{\sum_{j=1}^{n}\left|\frac{\partial G}{\partial y_{j}}\right|}}, i=1, . . n
$$

$\gamma_{i}$ est le coefficient reliant la valeur moyenne et l'écart type tel que $\gamma_{i}=\sigma_{i} / m_{i}$ et avec le signe \pm dépend du signe du dérivé:

$$
\frac{\partial G}{\partial y_{i}}>0 \rightarrow S_{f_{i}}>0 \text { et } \frac{\partial G}{\partial y_{i}}<0 \rightarrow S_{f_{i}}<0, i=1, . . n
$$




\section{iii- Calcul de la solution optimale}

Dans la dernière étape, pour déterminer la conception optimale, nous incluons les valeurs de facteurs de sécurité calculés dans la valeur de variables de conception.

\section{Conception fiable de treillis}

Nous abordons une optimisation mono-objective des structures en treillis comme présenté dans la Fig.5. On suppose que la structure est modélisée par des éléments barres soumis uniquement à des forces axiales. Les paramètres du matériau sont présentés dans le tableau 1 et sont respectivement: le module de Young $E$, le coefficient de Poisson $v$, le paramètre de durcissement $h$, la température de référence $T_{0}$, le limite élastique $R$, le paramètre d'échelle de température $\beta$ et la transformation maximale $\varepsilon_{l}$. Les paramètres géométriques utilisés sont indiqués dans le tableau 2 .

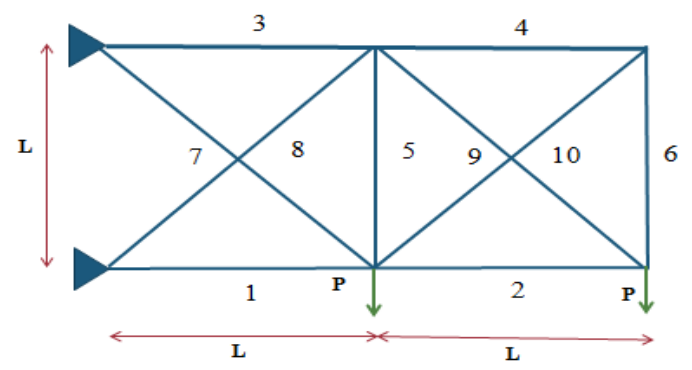

Fig. 5. Treillis en alliage à mémoire de forme

Tableau 1: Paramètres de matériau en alliage à mémoire de forme

\begin{tabular}{|c|c|c|c|c|c|c|c|}
\hline Paramètre & $E(M P a)$ & $v$ & $h(M P a)$ & $T_{0}(K)$ & $R(M P a)$ & $\beta\left(M^{2} a K^{-1}\right)$ & $\varepsilon_{l}$ \\
\hline Valeur & 51700 & 0.3 & 1000 & 248 & 140 & 5.6 & 0.1 \\
\hline
\end{tabular}

Tableau 2: Paramètres géométriques de treillis

\begin{tabular}{|c|c|c|c|c|}
\hline Paramètre & $L(\mathrm{~m})$ & $P(N)$ & $d_{i, 1} i=\{1, \ldots, 10\}(\mathrm{m})$ & $d_{i, 2} i=\{1, . ., 10\}(\mathrm{m})$ \\
\hline Signification & Longueur & Force axiale & Première dimension & Deuxième dimension \\
\hline Valeur & 9.144 & $4.45 .10^{5}$ & 0.0567 & 0.0567 \\
\hline
\end{tabular}

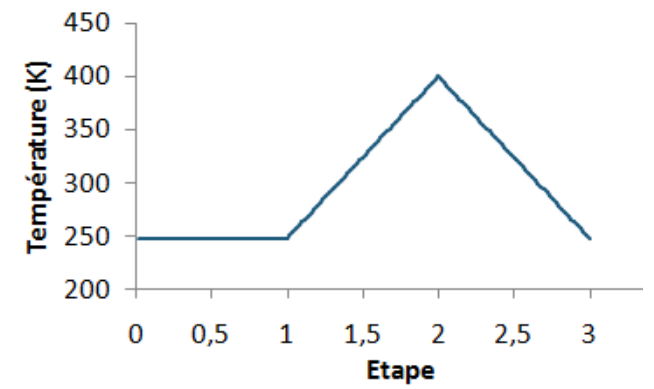

(a) Charges mécaniques

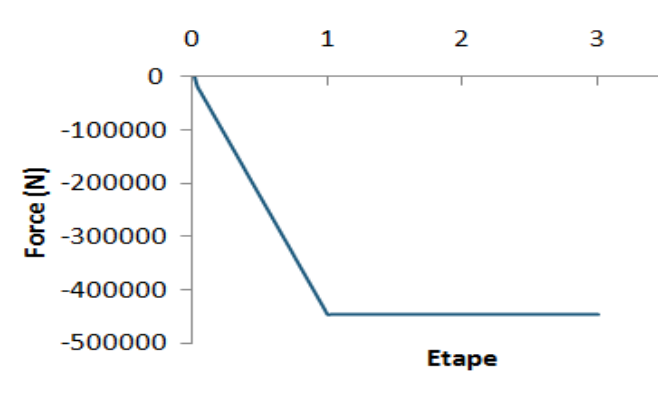

(b) Charges thermiques

Fig. 6. Chargements appliqués à la structure 
Fig.7 montre le déplacement transversal au cours des étapes 1, 2 et 3. Le déplacement augmente avec l'augmentation de la charge. Le déplacement diminue avec l'augmentation de la température de chauffage. Il semble que la structure commence à se déplacer à une température d'environ $296 \mathrm{~K}$. On peut observer que lorsque la température du chauffage atteint environ $315 \mathrm{~K}$, le déplacement sature à une valeur asymptotique. Cela correspond à une transformation complètement inverse (martensite en austénite). Pendant la température de refroidissement, le déplacement augmente, et il commence à partir d'une température d'environ $266 \mathrm{~K}$, Ceci représente la transformation de l'austénite en martensite. Nous pouvons conclure que le chargement thermique change la position de la structure bien que la charge mécanique ne soit pas supprimée.

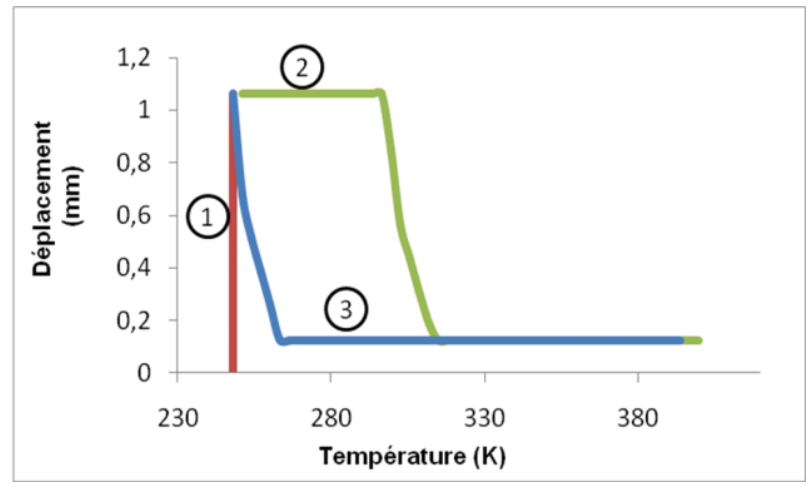

Fig. 7. Déplacement vertical de la structure en SMA

L'objectif de ce problème d'optimisation est de minimiser la masse structurelle de la treillis sans dépasser les contraintes admissibles. Le problème d'optimisation est défini par l'équation (12) tel que:

$$
\begin{aligned}
& \min w=\sum_{i=1}^{10} \rho \times A_{i} \times L_{i} \\
& \text { tel que }\left|\sigma_{j}\right|_{j=\{7, . ., 10\}} \leq \sigma_{\text {limite }}
\end{aligned}
$$

$\rho$ étant la densité du matériau, $L_{i}$ étant la longueur du i-ème élément et $A_{i}$ étant la section transversale du i-ème élément. $A_{i}$ peut être écrit comme le produit des deux dimensions de la section $d_{i, 1}$ et $d_{i, 2}$ avec $i=\{1, \ldots, 10\}$.

Les différents méthodes d'optimisation fiabiliste ont été appliqués. L'objectif de ce problème est de minimiser le poids $w$ de la structure sous contrainte mécanique $\sigma_{i}$ pour chaque élément diagonal. Nous notons que lorsque l'alliage à mémoire de forme est soumis à une contrainte très importante, la plasticité se produit après le domaine élastique de la martensite. Ensuite, une rupture est induite. Par conséquent, pour une analyse de fiabilité du matériau, la contrainte maximale ne doit pas dépasser une limite de charge $\sigma_{\text {limite }}=500$ MPa [21].

\section{Résultats et discussion}

L'objectif de cette section est de discuter les résultats des différentes méthodes d'optimisation. Le niveau de fiabilité cible $\beta^{T}$ est égale à 3 . En premier, on étudie la 
méthode d'optimisation déterministe (DDO). On concentre ensuite sur les méthodes d'optimisation fiabiliste. Le tableau 3 montre les résultats de la méthode DDO.

Tableau 3: Résultats de l'optimisation déterministe (DDO)

\begin{tabular}{|c|c|c|c|c|}
\hline & & $\begin{array}{c}\text { Valeurs } \\
\text { initiales } x_{0}\end{array}$ & $\begin{array}{c}\text { Point de } \\
\text { conception } y^{*}\end{array}$ & $\begin{array}{c}\text { Solution } \\
\text { optimale } x *\end{array}$ \\
\hline \multirow{8}{*}{$\begin{array}{l}\text { Variables de } \\
\text { conception }\end{array}$} & $d_{7,1}$ & 0.05 & 0.0263 & 0.0287 \\
\hline & $d_{7,2}$ & 0.05 & 0.0263 & 0.025 \\
\hline & $d_{8,1}$ & 0.05 & 0.025 & 0.025 \\
\hline & $d_{8,2}$ & 0.05 & 0.025 & 0.025 \\
\hline & $d_{9,1}$ & 0.03 & 0.0439 & 0.0574 \\
\hline & $d_{9,2}$ & 0.03 & 0.0439 & 0.0439 \\
\hline & $d_{10,1}$ & 0.03 & 0.025 & 0.0251 \\
\hline & $d_{10,2}$ & 0.03 & 0.025 & 0.025 \\
\hline $\begin{array}{l}\text { Fonction } \\
\text { objective }\end{array}$ & $w$ & 1704.8 & 1460.4 & 1512.2 \\
\hline \multirow{4}{*}{$\begin{array}{c}\text { Contraintes } \\
\text { d'optimisation }\end{array}$} & $\sigma_{7}$ & 57.0085 & 64.3132 & 57.3138 \\
\hline & $\sigma_{8}$ & 149.8332 & 495.1656 & 147.8543 \\
\hline & $\sigma_{9}$ & -2.0785 & -9.2829 & -2.0917 \\
\hline & $\sigma_{10}$ & 725.8643 & 500.7626 & 261.8870 \\
\hline $\begin{array}{l}\text { niveau de } \\
\text { fiabilité }\end{array}$ & $\beta$ & & & 2.55 \\
\hline
\end{tabular}

La solution optimale satisfait les contraintes des barres diagonales mentionnés précédemment. Cependant, le niveau de fiabilité est inférieur au niveau de fiabilité cible $\beta^{T}$. Après l'optimisation la structure, le poids résultant de la structure est $w_{D D O}=1512,2$ et l'indice de fiabilité de la solution optimale $\beta_{\text {DDO }}$ est égale à 2,55 qui est inférieure à $\beta^{T}$. On peut remarquer donc que la méthode classique présente une faible convergence à la stabilité. En conséquence, la méthode hybride (HM) est utilisée comme une méthode d'optimisation fiabiliste pour satisfaire le niveau de fiabilité requis. La méthode hybride est montrée dans le tableau 4.

Il est clair que le point optimal est dans le domaine de la défaillance $\left(\sigma_{10} \geq 500 \mathrm{MPa}\right)$ alors que la distance entre la conception point et le point optimal donne un niveau de fiabilité acceptable. Par conséquent, la méthode des facteurs de sûreté optimaux (OSF) semble être une technique attrayante qui peut surmonter les inconvénients.

Tableau 4: Résultats de la méthode hybride (HM)

\begin{tabular}{|c|c|c|c|c|c|}
\hline \multicolumn{2}{|c|}{} & $\begin{array}{c}\text { Valeurs } \\
\text { initiales } x_{0}\end{array}$ & $\begin{array}{c}\text { Valeurs } \\
\text { initiales } y_{0}\end{array}$ & $\begin{array}{c}\text { Point de } \\
\text { conception } y^{*}\end{array}$ & $\begin{array}{c}\text { Solution } \\
\text { optimale } x^{*}\end{array}$ \\
\hline \multirow{4}{*}{$\begin{array}{c}\text { Variables de } \\
\text { conception }\end{array}$} & $d_{7,1}$ & 0.05 & 0.05 & 0.0301 & 0.0303 \\
\cline { 2 - 6 } & $d_{7,2}$ & 0.05 & 0.05 & 0.0356 & 0.0301 \\
\cline { 2 - 6 } & $d_{8,1}$ & 0.05 & 0.05 & 0.034 & 0.0303 \\
\cline { 2 - 6 } & $d_{8,2}$ & 0.05 & 0.05 & 0.0356 & 0.0301 \\
\cline { 2 - 6 } & $d_{9,1}$ & 0.05 & 0.05 & 0.0333 & 0.0362 \\
\cline { 2 - 6 } & $d_{9,2}$ & 0.05 & 0.05 & 0.0358 & 0.0365 \\
\cline { 2 - 6 } & $d_{10,1}$ & 0.05 & 0.05 & 0.0329 & 0.0305 \\
\hline \multirow{2}{*}{$\begin{array}{c}\text { Fonction } \\
\text { objective }\end{array}$} & $d_{10,2}$ & 0.05 & 0.05 & 0.0324 & 0.0301 \\
\hline & $w$ & 1971.7 & 1971.7 & 1516.3 & 1476.6 \\
\hline
\end{tabular}




\begin{tabular}{|c|c|c|c|c|c|}
\hline \multirow[t]{3}{*}{ d'optimisation } & $\sigma_{8}$ & 147.8543 & 147.8543 & 311.6122 & 369.7436 \\
\hline & $\sigma_{9}$ & -2.0917 & -2.0917 & -4.9115 & -6.4204 \\
\hline & $\overline{\sigma_{10}}$ & 261.8870 & 261.8870 & 539.3570 & 576.3510 \\
\hline $\begin{array}{l}\text { niveau de } \\
\text { fiabilité }\end{array}$ & $\beta$ & & & & 3.16 \\
\hline
\end{tabular}

Le tableau 5 montre la différence de la stratégie OSF par rapport à la méthode DDO et HM. On voit clairement que la solution optimale satisfait les différentes contraintes. En fait, cette méthodologie satisfait le niveau de fiabilité requis mais donne une masse structurelle assez grande que celle obtenue par DDO de 2,41\%. Cependant, il est évident que le DDO peut conduire à des niveaux de fiabilité faible. Donc, on ne peut pas contrôler les exigences de fiabilité, mais en utilisant l'OSF, on obtient le compromis optimal entre la fonction objective et les différents contraintes appliqués à la structure .

Tableau 5: Résultats de la méthode des facteurs de sûreté optimaux (OSF)

\begin{tabular}{|c|c|c|c|c|c|}
\hline \multicolumn{2}{|c|}{} & $\begin{array}{c}\text { Valeurs } \\
\text { initiales } x_{0}\end{array}$ & $\begin{array}{c}\text { Point de } \\
\text { conception } y^{*}\end{array}$ & OSF $S_{f}$ & $\begin{array}{c}\text { Solution } \\
\text { optimale } x^{*}\end{array}$ \\
\hline \multirow{4}{*}{$\begin{array}{c}\text { Variables de } \\
\text { conception }\end{array}$} & $d_{7,1}$ & 0.05 & 0.0263 & 1.0077 & 0.0261 \\
\cline { 2 - 6 } & $d_{7,2}$ & 0.05 & 0.0263 & 1.0079 & 0.0261 \\
\cline { 2 - 6 } & $d_{8,1}$ & 0.05 & 0.025 & 1.0094 & 0.0248 \\
\cline { 2 - 6 } & $d_{8,2}$ & 0.05 & 0.025 & 1.0095 & 0.0248 \\
\cline { 2 - 6 } & $d_{9,1}$ & 0.03 & 0.0439 & 0.8271 & 0.0531 \\
\cline { 2 - 6 } & $d_{9,2}$ & 0.03 & 0.0439 & 0.8269 & 0.0531 \\
\cline { 2 - 6 } & $d_{10,1}$ & 0.03 & 0.025 & 0.8766 & 0.0285 \\
\hline \multirow{2}{*}{$\begin{array}{c}\text { Fonction } \\
\text { objective }\end{array}$} & $d_{10,2}$ & 0.03 & 0.025 & 0.0876 & 0.0285 \\
\hline \multirow{3}{*}{$\begin{array}{c}\text { Contraintes } \\
\text { d'optimisation }\end{array}$} & $\sigma_{7}$ & 1704.8 & 1460.4 & & 1548.7 \\
\cline { 2 - 6 } & $\sigma_{8}$ & 149.8832 & 495.1656 & & 64.7381 \\
\cline { 2 - 6 } & $\sigma_{9}$ & -2.0785 & -9.2829 & & -9.4348 \\
\hline \multirow{2}{*}{$\begin{array}{c}\text { niveau de } \\
\text { fiabilité }\end{array}$} & $\beta$ & 725.8643 & 500.7626 & & 344.2413 \\
\hline \multirow{2}{*}{} & \multicolumn{3}{|l}{} & & 3 \\
\hline
\end{tabular}

\section{Conclusion}

Dans ce papier, l'optimisation de treillis en alliage à mémoire de forme (SMA) a été réalisée en tenant compte des incertitudes dans les dimensions de la structure. les différentes méthodes d'optimisation fiabilistes ont été développé afin d'obtenir une conception optimal qui garantit le niveau de fiabilité requis. On a montré que l'optimisation déterministe (DDO) est facile à mettre en œuvre. Cependant, le niveau de fiabilité n'est pas contrôlé. Ensuite, on a trouvé que lorsque la méthode hybride (HM) est appliquée, le point de conception est dans la zone de défaillance donc le résultat est rejeté. Pour surmonter ce problème, on a utilisé la méthode des facteurs de sûreté optimaux (OSF). Bien que le modèle mécanique utilisé est assez simple, la méthodologie d'optimisation fiabiliste peut être étendue à des structures plus complexes. De plus, les méthodes d'optimisation décrites précédemment sont limitées dans le cas où seules les variables de conception peuvent être traitées comme des variables aléatoires. Afin de surpasser une telle limitation, plus de recherche est nécessaire. 


\section{Remerciement}

Ce travail de recherche a été soutenu par le Laboratoire de Mécanique de Normandie (LMN), l'Institut National des Sciences Appliquées de Rouen et le Laboratoire de Mécanique, Modélisation et Productique (LA2MP), l'Ecole Nationale d'Ingénieurs de Sfax. Les auteurs remercient le soutien de ces institutions.

\section{Références}

1. A. Alipour, M. Kadkhodaei, A. Ghaei, Finite element simulation of shape memory alloy wires using a user material subroutine: Parametric study on heating rate, conductivity, and heat convection, Journal of Intelligent Material Systems and Structures, 26(5), 554-572 (2015)

2. T. Merzouki, A. Duval, T. B. Zineb, Finite element analysis of a shape memory alloy actuator for a micropump. Simulation Modelling Practice and Theory, 27, 112-126 (2012)

3. D. C. Lagoudas ed. Shape memory alloys: modeling and engineering applications. Springer Science and Business Media, 2008.

4. R. Croquet, D. Lemosse, E. Souza De Cursi, Y. Aoues, A. El Hami. Un schéma itératif pour l'optimisation fiabiliste basé sur l'obtention de coefficients de sécurités optimaux. 20ème Congrès Français de Mécanique, Besançon, France, 28 août $/ 2$ sept. 2011

5. A. Yaich, G. Kharmanda, A. El Hami, L. Walha, M. Haddar, Reliability Based Design Optimization for Multiaxial Fatigue Damage Analysis Using Robust Hybrid Method. Journal of Mechanics, 1-16 (2017)

6. R. Grandhi, L. Wang, Reliability-based structural optimization using improved towpoint adaptive nonlinear approximations, Finite Elem Anal Des, 29, 35-48, 1998

7. G. Kharmanda, S. Sharabatey, H. Ibrahim, A. Makhloufi, A. Elhami, Reliability-Based Design Optimization using Semi-Numerical Strategies for Structural Engineering Applications. International Journal of CAD/CAM, 9(1), 1-16 (2009)

8. G. Kharmanda, A. Mohamed, M. Lemaire, Efficient reliability-based design optimization using a hybrid space with application to finite element analysis. Structural and Multidisciplinary Optimization, 24(3), 233-245 (2002)

9. K. Dammak, A. El Hami, , S. Koubaa, L. Walha, M. Haddar, Reliability based design optimization of coupled acoustic-structure system using generalized polynomial chaos. International Journal of Mechanical Sciences, 134, 75-84 (2017)

10. I. Kusano, A. Baldomir, J. A. Jurado, S. Hernández, Reliability based design optimization of long-span bridges considering flutter, Journal of Wind Engineering and Industrial Aerodynamics, 135, 149-162 (2014)

11. A. Makhloufi, Y. Aoues, A. El Hami, Reliability based design optimization of wire bonding in power micro-electronic devices, Microsystem Technologies, 22, 2737-2748 (2016)

12. R. El Maani, A. Makhloufi, B. Radi, A. El Hami, Reliability-based design optimization with frequency constraints using a new safest point approach, Engineering Optimization, 1-18, 2018

13. G. Kharmanda et A. El Hami, Reliability in Biomechanics, UK: Wiley \& ISTE, 2016. 
14. G. Kharmanda, Numerical and semi-numerical methods for reliability-based design optimization, Structural design optimization considering uncertainties, Taylor \& Francis e-Library, 189-216 (2008)

15. G. Kharmanda, M. Ibrahim, A. Abo Al-kheer, F. Guerin, A. El-Hami, Reliabilitybased design optimization of shank chisel plough using optimum safety factor strategy, Computers and Electronics in Agriculture, 109, 162-171 (2014)

16. Y. Aoues, A. Chateauneuf, Benchmark study of numerical methods for reliabilitybased design optimization, Struct Multidisc Optim, 41, 277-294, (2010)

17. G. Kharmanda, N. Olhoff, A. El-Hami, Optimum values of structural safety factors for a predefined reliability level with extension to multiple limit states, Structural and Multidisciplinary Optimization, 27, 421-434 (2004)

18. G. Kharmanda, N. Olhoff, , Extension of optimum safety factor method to nonlinear reliability-based design optimization, Journal of Structural and Multidisciplinary Optimization, 43, 367-380 (2007)

19. R. H Lopez, D. Lemosse, E. S. Cursi, J. E. Rojas, A. El-Hami, An Approach for the Reliability Based Design Optimization of Laminated Composite Plates, Engineering Optimization, 43, 1079-1094 (2011)

20. B. Xia, D. Yu, Optimization Based on reliability and confidence interval design for the structural-acoustic system with interval probabilistic variables, Journal of sound and vibration, 336, pp. 1-15, (2014)

21. W. Huang, Modified shape memory alloy (SMA) model for SMA wire based actuator design, Journal of intelligent material systems and structures, 10(3), 221-231 (1999) 